Classic diseases revisited

\title{
Superior mesenteric artery syndrome
}

\author{
AR Ahmed, I Taylor
}

\begin{abstract}
Summary
Superior mesenteric artery syndrome is a rare and controversial form of upper intestinal obstruction in which the third part of the duodenum is compressed by the overlying superior mesenteric artery. Any disease process decreasing the angle between the superior mesenteric artery and the abdominal aorta can result in the external compression of the duodenum and subsequent intestinal obstruction. The aetiology, presentation, investigation and management of this unusual condition are discussed.
\end{abstract}

Keywords: superior mesenteric artery syndrome, intestinal obstruction

Department of Surgery, University College London Medical School, 67-73 Riding House St, London W1P 7LD, UK

AR Ahmed

I Taylor

Correspondence to Professor I Taylor

Accepted 10 February 1997
Rokitansky is generally credited with the earliest description (in 1861) of the compression of the third part of the duodenum by the superior mesenteric artery (SMA). ${ }^{1}$ Surprisingly, there was little interest in the subject until 1927, when Wilkie reported seven instances of superior mesenteric artery syndrome (SMAS). ${ }^{2}$ Since then, this entity has remained a subject of controversy. The signs and symptoms characterising the syndrome were not regarded as unique. Many observers considered them to be mere manifestations of another abdominal process ${ }^{3-7}$ and remained unconvinced of the reality of the syndrome. They regarded the role of the SMA in producing duodenal obstruction as circumstantial, especially as in many of the reported cases, the obstruction was later found to be due to neoplastic or inflammatory disease, or electrolyte imbalance.

The advent of modern radiological techniques in the 1960 s provided workers with the evidence needed to support the existence of SMAS, ${ }^{8}$ although the skeptics persisted in their view, despite clear evidence of the actuality of the syndrome. Over the years, SMAS has also received other names, such as aortomesenteric duodenal compression, Wilkie's syndrome, Cast syndrome and chronic duodenal ileus.

\section{Aetiology}

One of the consequences of the erect posture of humans is that the superior mesenteric artery leaves the aorta at an acute downward angle, rather than at a nearly complete right angle as it does in quadrupeds. It is through this vascular angle, between the aorta and the SMA, that the third part of the duodenum passes (figure 1), ${ }^{9}$ and is thus vulnerable to becoming pinched in between the SMA anteriorly and the aorta and vertebral column posteriorly. A number of factors may predispose to this vascular compression of the duodenum.

The duodenum is suspended in the angle by the ligament of Treitz. This suspensory ligament's site of attachment and structure ${ }^{10}$ may differ from individual to individual. In most people, the duodenum crosses the vertebral column at the level of the third lumbar vertebra, but a short suspensory ligament may raise it, bringing the duodenum higher into the vascular angle between the SMA and the aorta. A lower duodenal position may not be advantageous either, as the spine curves anteriorly at LA thus reducing the width of the gap between SMA and aorta at that level.

In a normal person, the mass of fat and lymphatic tissue around the origin of the SMA provides adequate protection to the duodenum against compression. However, it has been postulated that if a patient loses weight rapidly, for example, as a sequelae to debilitating injury ${ }^{11}$ or an eating disorder, ${ }^{12}$ or, indeed, gains height rapidly without a concomitant increase in weight, the fatty cushion around the SMA diminishes. ${ }^{13}$ Consequently there is a decrease in the angle between the SMA and the aorta from the usual $45^{\circ}$ down to $15^{\circ}$, resulting in occlusion of the third part of the duodenum.

Other factors that may narrow this vascular angle and contribute to the development of SMAS include increased lordosis and external pressure such as the application of a body cast in the treatment of scoliosis or vertebral fracture. ${ }^{14}$ Recent reports also describe SMAS as a rare postoperative complication following total proctocolectomy and ileal pouch anastomosis, ${ }^{15,16}$ in abdominal aortic aneurysm ${ }^{17}$ or following repair of the latter, ${ }^{18}$ with Strongyloides stercoralis infection, ${ }^{19}$ in hyperthyroidism, ${ }^{20}$ and even during pregnancy. ${ }^{21}$

Choi and Pfalzer $^{22}$ have suggested that the critical factor is not the narrow aorto-mesenteric angle and distance alone but the course of the SMA running anterior and parallel to the aorta. Others have refuted the theory of vascular compression and suggested that the compression of the duodenum is caused by peritoneal adhesions which are a result of duodenal malrotation ${ }^{23-25}$ or inflammatory thickening of the mesenteric root secondary to acute pancreatitis, a duodenal ulcer or infarction of the bowel. ${ }^{26}$ 


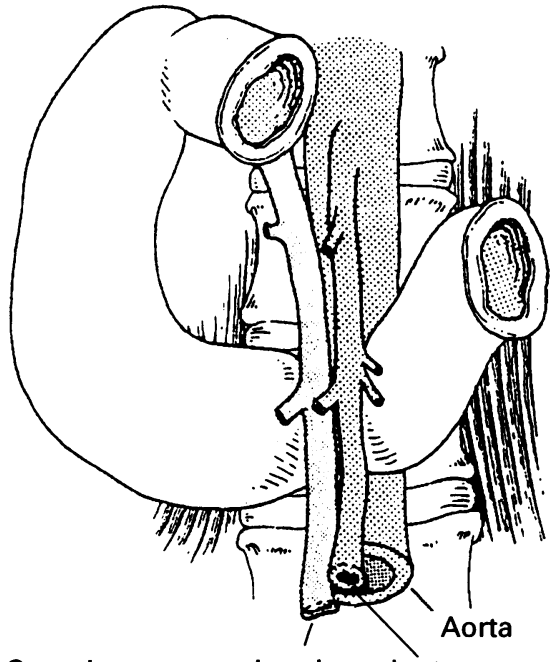

Superior mesenteric vein and artery

A

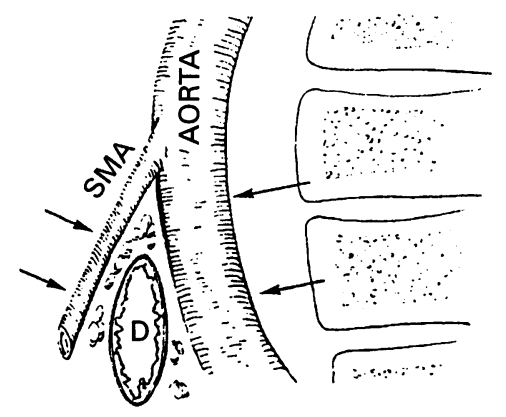

B

Figure 1 (A) Diagram of the anatomy of the third part of the duodenum and (B) its relationship to the aorta, vertebrae and superior mesenteric vessels (from Jones PA, Wastell C. Superior mesenteric artery syndrome. Postgrad Med F 1983; 59:376-9)

\section{Superior mesenteric artery} syndrome

Predisposing factors

- abnormally acute aortomesenteric vascular angle with a short SMA aorta gap

- abnormally high fixation of duodenojejunal flexure to the ligament of Treitz

- exaggerated lumbar lordosis

- unusually low origin of the superior mesenteric artery

- supine position

Precipitating factors

- marked weight loss with subsequent loss of mesenteric and retroperitoneal fat as may occur in severe wasting diseases such as cancer and burns, severe injuries such as head trauma, dietary disorders such as anorexia nervosa or malabsorption, postoperative state disease, deformity, or trauma to the spine, including application of a body cast
The SMAS should be distinguished from a SMA-like syndrome. Sometimes, duodenal dilatation proximal to compression by the SMA may essentially be part of generalised dilatation of duodenum of variable aetiology and not secondary to obstruction by a compressing structure. Gondos ${ }^{27}$ studied 11 cases of systemic sclerosis where a compression defect of the duodenum was found at the site where the SMA crossed the duodenum. All 11 patients were found to have definite dilatation of the duodenum - a result of atrophy of the muscle layers and replacement by collagenous tissue. These changes cause diminished peristalsis, loss of muscle tone, and dilatation which renders the duodenum vulnerable to a concave pressure defect in its third portion in response to a prominent structure - the SMA. Gondos concludes that the compression in such cases is brought on by imprinting by the SMA, although this occurs only secondarily as a response to dilatation and loss of muscle tone of the duodenum. A recent case report of duodenal compression defect in a patient suffering from mixed connective tissue disease also illustrates this point. ${ }^{28}$ Indeed, several pathologic conditions may predispose to the development of a megaduodenum that may result in an imprint on the duodenum at the level of the SMA. The most notable of these conditions is scleroderma ${ }^{27}$; however, other causes of reduced duodenal peristalsis such as diabetes, pancreatitis, dermatomyositis, lupus erythematosus, myxoedema, amyloidosis, myotonic dystrophy, or chronic idiopathic intestinal pseudo-obstruction can cause this SMA-like syndrome. ${ }^{29,30}$ Some of the predisposing and precipitating factors in SMAS are summarised in the box.

\section{Clinical features}

Patients may present acutely or with a chronic, insidious history. Acutely, patients usually present with symptoms and signs of duodenal obstruction, namely, nausea, vomiting, abdominal pain, distension, tympani, tenderness and abnormal bowel sounds. These symptoms are aggravated by eating. They are, however, classically relieved with postural change in particular the knee-chest or prone positions, both of which increase the angle between the SMA and aorta. Manifestations of electrolyte imbalance may occur. ${ }^{31}$

The chronic cases may present many times over many years for investigation of intermittent abdominal pain associated with vomiting, early satiety and anorexia.

\section{Diagnosis}

Plain abdominal X-ray demonstrates gastric dilatation. Endoscopic examination usually does not indicate the diagnosis. On barium meal, a positive diagnosis of SMAS can be made in the presence of duodenal dilatation, retention of barium within the duodenum and the characteristic vertical linear extrinsic pressure in the third portion of the duodenum. The dilated segment proximal to the obstruction may show reverse peristalsis. Hayes manoeuvre is the demonstration of the disappearance of these radiological features in the knee-chest position on cinefluoroscopy. ${ }^{32}$ Hypotonic duodenography combined with simultaneous SMA arteriography has been noted as being a more accurate investigation than a routine barium meal examination. ${ }^{32,33}$ More recently, computed tomography (CT) has proved successful in providing diagnostic insight into cases of SMAS. ${ }^{34} \mathrm{CT}$ was able to demonstrate simultaneously the characteristic duodenal distension and the close proximity of superior mesenteric vessels and aorta. Its advantages include that it is a safe, rapid and relatively noninvasive technique.

\section{Treatment}

The condition may be managed conservatively, though surgical treatment is sometimes required, perhaps partly to establish the diagnosis.

\section{CONSERVATIVE MANAGEMENT}

Gastric dilatation occurs early in vascular compression of the duodenum and nasogastric suction accompanied by alterations in posture such as placing the patient into the prone position may abort full-blown duodenal obstruction. After gastric decompression, restoring retroperitoneal fat by giving multiple small feeds peroral in the left lateral or prone position has been effective in relieving the obstruction. ${ }^{35}$ Others favour aggressive nutritional support using nasojejunal feeding past the point of obstruction as the primary method of nutrition support. ${ }^{36}$ Alternatively, total parenteral nutrition or a combination of enteral and parenteral feeding may be necessary to meet nutritional needs until 


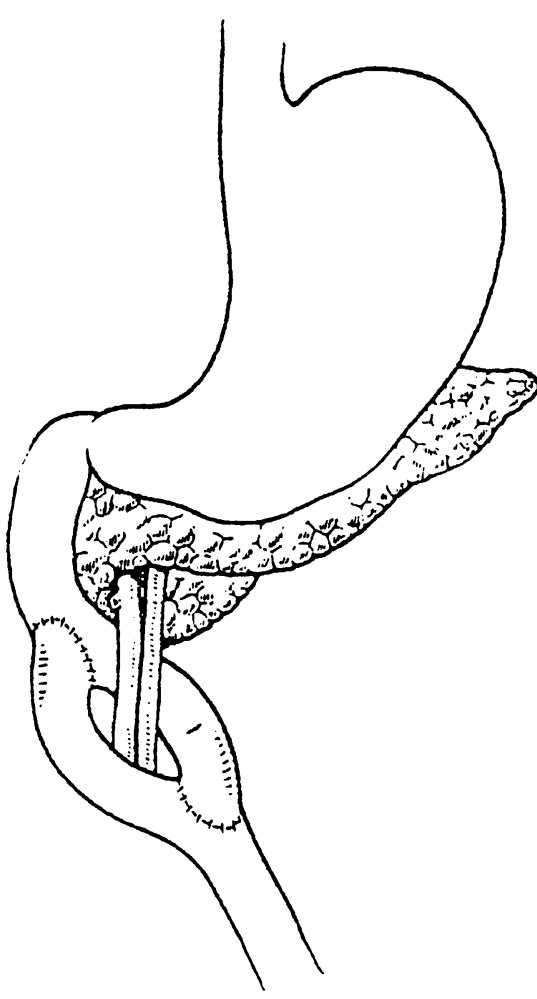

Figure 2 Duodenojejunostomy (from Jones PA, Wastell C. Superior mesenteric artery syndrome. Postgrad Med $\mathcal{F}$ 1983; 59:376-9)

1 Rokitansky CV. Lehrbuch der patologische Anatomie, 3rd edn, vol 3. Vienna: Braumuller, 1861; p 187

2 Wilkie DPD. Chronic duodenal ileus. Am $\mathcal{F}$ Med Sci 1927; 173: 643 .

3 Cimmino CV. Arteriomesenteric occlusion of the duodenum: an entity? Radiology 1961; 76: 828-9.

4 Cimmino CV. The status of the syndrome of arteriomesenteric occlusion of the duodenum. A critical review. Virginia Med Monthly 1961; 88: $192-204$.

5 Dorph MH. The Cast syndrome. Review of the literature and report of a case. $N \mathrm{Engl} f \mathrm{Med}$ 1950; 243: $440-2$.

6 Dragstedt LR, Dragstedt CA. Acute dilatation of the stomach. $₹ A M A$ 1922; 79: $612-5$.

7 Eaton SB Jr, Ferrucci JT Jr. Radiology of the pancreas and duodenum. Philadelphia: Saunders, 1973.

8 Anderson JR. Extrinsic compression of the third part of the duodenum. Clin Radiol 1982; 33: $75-81$.

9 Akin ST, Skandalakis JE, Gray SW. The anatomic basis of vascular compression of the duodenum. Surg Clin North Am 1974; 54: $1361-70$.

10 Akin JT. Vascular compression of the duodenum. Surgery 1976; 79: 512-22.

11 Roth EJ, Fenton LL, Gaebler-Spira DJ, Frost FS, Yarkong GM. Superior mesenteric artery syndrome in acute traumatic quadraplegia: case reports and literature review. Arch Phys Med Rehabil 1991; 72: 417-20.

12 Pentlow BD. Acute vascular compression of the duodenum in anorexia nervosa. Br F Surg 1981; 68: $665-6$.

13 Hines JR, Gore RM, Ballantyne GH. Superior mesenteric artery syndrome: diagnostic criteria 148: $630-2$.

14 Berk RN, Coulson DB. The Cast Syndrome. Radiology 1970; 94: 303-3.

15 Ballantyne GH, Graham SM, Hammers L, Modlin IM. Superior mesenteric artery syndrome following ileal J-pouch anal anastomosis. An iatrogenic cause of early postoperative obstruction. Dis Colon Rectum 1987; 30: 472-4. the duodenal obstruction resolves, the aim being to supply sufficient calories to increase mesenteric fat and expand the angle of the mesenteric root.

Occasionally, treatment with metoclopramide intravenously and later orally has facilitated gastroduodenal emptying. ${ }^{34}$

\section{SURGICAL MANAGEMENT}

For patients in whom conservative methods fail, several surgical procedures have been described. Strong's procedure ${ }^{37}$ which mobilises the duodenum by dividing the ligament of Treitz has the benefit of not requiring anastomosis of the bowel. But, on its own, Louw ${ }^{38}$ claims this produces an unacceptably high failure rate and recommends division of this ligament with complete mobilisation of the duodenum followed by passage of most of the jejunum and ileum underneath the superior mesenteric vessels into the right side of the abdomen. The ascending colon is then separated from its retroperitoneal attachments and placed in the left side of the abdomen. The end result is a configuration similar to that seen in congenital malrotation of the intestine.

When intraperitoneal adhesions make Strong's operation difficult, duodenojejunostomy, first described by Staveley, ${ }^{39}$ is the preferred operation (figure 2). This method has been favoured over the years and the vast majority of results have been excellent. Occasionally, merely emptying the stomach by gastrotomy will relieve the situation, particularly if associated with full mobilisation of the duodenum. In patients in whom gastric distension dominates the clinical picture and when the other procedures are technically difficult, gastrojejunostomy can provide the needed drainage. Consideration should be given to a vagotomy when performing the latter procedure because of the risk of marginal ulceration. ${ }^{40}$ Transposition of the third part of the duodenum anteriorly to the superior mesenteric vessels has also been successful and aims at permanently circumventing the obstruction. ${ }^{41}$

16 Goes RN, Coy CS, Amaral CA, Fagundes JJ, Medeiros RR. Superior mesenteric artery syn-
drome as a complication of ileal pouch - anal drome as a complication of ileal pouch-anal
anastomosis. Dis Colon Rectum 1995; 38: 543-4. 17 Edwards KC, Katzan BT. Superior mesenteric artery syndrome due to large dissecting abdominal aortic aneurysm. Am $\mathcal{F}$ Gastroenterol 1984; 79: $72-4$

18 Lamont PM, Clarke PJ, Collin J. Duodenal obstruction after abdominal aortic aneurysm. Eur $\mathcal{F}$ Vasc Surg 1992; 6: $107-10$.

19 Lee MJ, Terry SI. Aortomesenteric duodenal occlusion associated with Strongyloidiasis. $f$ Trop Med Hyg 1989; 92: 41-5.

20 McClenathan JH, Wood BP. Radiological case of the month: hyperthyroidism as a cause of of the month: hyperthyroidism as a cause of
superior mesenteric artery syndrome. $A m \mathcal{F}$ Dis superior mesenteric artery

21 Iko B, Mondu JU, Orhue A, Sarkar SK. The superior mesenteric artery syndrome in pregnancy: a case resulting in recurrent pregnancy loss. Eur f Obstet Gynecol Reprod Biol 1986; 21: 233-6.

22 Choi H, Pfalzer FA. Superior mesenteric artery syndrome. NY State $\mathcal{f}$ Med 1976; 76: $986-8$.

23 Lauge - Hansen N. Developmental and embryonic anatomy from the $15 \mathrm{~mm}$ stage to the end of gestation - personal investigation. In: Lauge Hansen $\mathrm{N}$, ed, Developmental anatomy of the Hansen $\mathrm{N}$, ed, Developmental anatomy of the
human gastrointestinal tract. Copenhagen: human gastrointestinal tract.

24 Grawgeard E. Malrotation of the duodenum. In: Lauge-Hansen N, ed, Developmental anomalies of the gastrointestinal tract due to malrotation Copenhagen: Munksgaard, 1974; pp 112-8.

25 Wayne ER, Burrington JD. Duodenal obstruc tion by the superior mesenteric artery in children. Surgery 1978; 72: 762-8.

26 Simon M, Lerner MA. Duodenal compression by the mesenteric root in acute pancreatitis and inflammatory conditions of the bowel. Radiology 1962; 79: 75-80.

27 Gondos B. Duodenal compression defect and the 'superior mesenteric artery syndrome'. Radithe 'superior mesenteric a
ology 1977; 123: 575-80

28 Ahmed AR, Taylor I. The superior mesenteric artery syndrome in mixed connective tissue disease. Gastroenterol Today 1997; in press.
29 Cohen LB, Field SP, Sachar AP. The superior mesenteric artery syndrome. The disease that
isn't, or is it? $\mathcal{F}$ Clin Gastroenterol 1985; 7: 113-6. 30 Eisenberg RL. Gastroenterology: a pattern approach. Philadelphia: JB Lippincott, 1983; pp $403-10$.

31 Lundell L, Thulin A. Wilkie's syndrome: a rarity? Br f Surg 1980; 67: 604-7.

32 Lukes PJ, Rolny P, Nilsson AE, et al. Diagnostic value of hypotonic duodenography in superior mesenteric artery syndrome. Acta Chir Scand 1978; 144: 39-43.

33 Gustafsson L, Falk A, Lukes PJ, Ganklou R. Diagnosis and treatment of superior mesenteric Diagnosis and treatment of superior mesenteric

34 Applegate GR, Cohen AJ. Dynamic CT in Applegate GR, Cohen AJ. Dynamic CT in
superior mesenteric artery syndrome. $f$ Comput Assist Tomogr 1988; 12: $976-80$ Superior mesenteric artery syndrome in traumatic brain injury: two cases. Arch Phys Med Rehabil 1995; 76: 871-5.

36 Milner EA, Cioffi WG, McManus WF, Pruitt

37 Strong EK. Mechanics of aortomesenteric duodenal obstruction and direct surgical attack upon aetiology. Ann Surg 1958; 148: 725-30.

38 Louw JH. Chronic duodenal ileus. $₹ R$ Coll Surg Edin 1959; 5: 101-26.

39 Staveley AL. Chronic gastromesenteric ileus. Surg Gynecol Obstet 1910; 11: 288-91.

40 Barner HB, Sherman CD. Vascular compression of the duodenum. Int Abstracts Surg 1963; 117: $103-18$.

41 Duvie SO. Anterior transposition of the third part of the duodenum in the management of chronic duodenal compression by the superior mesenteric artery. Int Surg 1988; 73: 140-3.
35 Pedoto MJ, O'Dell MW, Thrun M, Hollifield D. BA. Superior mesenteric artery syndrome in a burn patient. Nutr Clin Pract 1993; 8: 264-6. 